\title{
Usos tradicionais e propriedades fitoterápicas do dente-de-leão (Taraxacum officinale F.H. Wigg.)
}

\author{
Traditional use and herbal properties of dandelion (Taraxacum officinale F.H. Wigg.)
}

Usos tradicionales y propiedades herbales del diente de león (Taraxacum officinale F.H. Wigg.)

Gabriela Mota Silva de Oliveira ${ }^{1 *}$, Bruna da Silva de Brito ${ }^{1}$, Fernanda Oliveira de Gaspari de Gaspi .

\section{RESUMO}

Objetivo: Fornecer uma revisão integrativa da literatura abrangendo estudos com base em evidências com experimentos in vivo e in vitro para avaliação dos efeitos fitoterápicos do dente-de-leão, que buscaram comprovar os seus usos tradicionais e propriedades fitoterápicas. Métodos: Trata-se de uma revisão sistêmica abrangendo estudos com base em evidências com experimentos in vivo e in vitro para avaliação dos efeitos fitoterápicos do dente-de-leão, que buscaram comprovar os seus usos tradicionais além da sua etnofarmacologia presente na literatura. Resultados: Os experimentos utilizados para avaliação da funcionalidade do dente-de-leão, tanto in vivo quanto in vitro obtiveram resultados satisfatórios durante a avaliação, comprovando a eficácia do dente-de-leão sob diversas patologias e afirmando seus descritos na etnofarmacologia, além da comprovação dos seus usos tradicionais conhecidos. Conclusão: Conclui-se que os usos tradicionais e as propriedades fitoterápicas do dente-de-leão são eficazes. Os estudos utilizados nesta revisão atingiram seus objetivos com êxito, nos mostrando a importância de novos estudos sobre a Taraxacum officinale.

Palavras-chave: Etnofarmacologia, Fitoterapia, Taraxacum officinale.

\begin{abstract}
Objective: Provide an integrative review of the literature covering evidence-based studies with in vivo and in vitro experiments to assess the effects herbal medicines of the dandelion, who sought to prove their traditional uses and herbal properties. Methods: This is a systemic review covering evidence-based studies with in vivo and in vitro experiments to assess the effects of herbal effects of the dandelion, which sought to prove its traditional uses beyond its ethnopharmacology present in the literature. Results: The experiments used to evaluate the functionality of the dandelion, both in vivo and in vitro, obtained satisfactory results during the evaluation, proving the efficacy of dandelion under various pathologies and stating its described in ethnopharmacology proof of its known traditional uses. Conclusion: It is concluded that traditional uses and herbal properties of dandelion are effective. The studies used in this review have successfully achieved their objectives, showing us the importance of further studies on Taraxacum officinale.
\end{abstract}

Keywords: Ethnopharmacology, Phytotherapy, Taraxacum officinale.

\section{RESUMEN}

Objetivo: Proporcione una revisión bibliográfica integradora que abarque estudios basados en evidencia con experimentos in vivo e in vitro para evaluar los efectos fitoterapéuticos del diente de león, que intentaron probar sus usos tradicionales y propiedades fitoterapéuticas. Métodos: Esta es una revisión sistémica que abarca estudios basados en evidencia con experimentos in vivo e in vitro para evaluar los efectos fitoterapéuticos del diente de león, que buscaban probar sus usos tradicionales además de su etnofarmacología presente en la literatura. Resultados: Los experimentos utilizados para evaluar la funcionalidad del diente de león, tanto in vivo como in vitro, obtuvieron resultados satisfactorios durante la evaluación, demostrando la eficacia del diente de león bajo diversas patologías y afirmando su descripción en etnofarmacología, además de confirmar los resultados. sus usos tradicionales conocidos. Conclusión: Se

${ }^{1}$ Fundação Hermínio Ometto (UNIARARAS), Araras - SP. *E-mail: gabismotaa@gmail.com 
concluye que los usos tradicionales y las propiedades herbales del diente de león son efectivos. Los estudios utilizados en esta revisión lograron con éxito sus objetivos, lo que nos muestra la importancia de realizar más estudios sobre Taraxacum officinale.

Palabras clave: Etnofarmacología, Medicina herbaria, Taraxacum officinale.

\section{INTRODUÇÃO}

As plantas medicinais estão relacionadas com a sabedoria popular, e embora algumas delas tenham sido esquecidas no decorrer dos anos devido às novas tecnologias, elas ainda possuem grandes fontes de aplicações e estudos, como é o caso da espécie Taraxacum officinale F.H. Wigg., que pertence à família Asteraceae e apresenta grande importância em relação à ação farmacológica e como alimento, sendo fonte de vários princípios ativos e substâncias de valor alimentício, como vitaminas e sais minerais (ROQUE $\mathrm{N}$ e BAUTISTA H, 2008). A planta é popularmente conhecida como Dente-de-Leão e Amargosa (RIBEIRO M, et al., 2004).

A planta é originária da Europa, mostra-se como uma espécie ruderal com ampla distribuição geográfica. Apresenta raiz bem desenvolvida, são herbáceas, apresentando caules macios e flexíveis e folhas amarelas, podendo ser cultivada em diversos solos, instalando-se em gramados, jardins, hortas e lavouras (RIBEIRO $\mathrm{M}$, et al., 2004). A localização desta planta não é predominante em apenas determinada região, sendo assim, possibilita a utilização da mesma por diversos povos de diferentes culturas. Independente da etnia ou estatuto social da população que utiliza a manipulação e preparação para o consumo e utilização não sofrem alterações relevantes, obtendo mesmo resultado se comparado entre os continentes (MARTINEZ M, et. al., 2015).

O dente-de-leão é utilizado tradicionalmente de forma crua, em saladas ou seco para preparação de infusões ou cozido. Praticamente todas as suas partes são utilizadas, folhas, flores, raizes. Além disso, a raiz pode ser torrada para a utilização como substituto do café (MARTINEZ M, et al., 2015). As atribuições terapêuticas são diversas, as quais surgiram principalmente devido aos seus usos tradicionais. Por ter demonstrado resultados positivos, gerou replicação de informação passando de geração em geração.

A partir do século XV, a planta Taraxacum officinale foi reconhecida como droga vegetal para o tratamento de transtornos hepáticos. A mesma apresenta em sua composição vitamina $A$, que auxilia no sistema imunológico e vitamina $\mathrm{B}$, importante para o metabolismo do organismo. Além disso, a planta é rica em ferro, cobre e potássio (RIBEIRO M, et al., 2004). A atividade antioxidante desta planta é de extrema importância para a preservação e manutenção da saúde e o estado nutricional da população, sendo indispensáveis para a defesa do organismo (TURECK C, et al., 2017).

O dente de leão apresenta inúmeros benefícios em relação às propriedades nutricionais, possuindo em sua composição uma grande quantidade de água, flavonoides, minerais e vitaminas, auxiliando e prevenindo supostas patologias (GUIRACOCHA JCM, 2014). Suas propriedades fitoterápicas aumentaram depois da origem dos novos conhecimentos, além de ser rica em ferro obtendo função de proteger células hepáticas, auxilia no tratamento de problemas dermatológicos, e atividade anti-inflamatória (RIGOBERTO JVH, 2017), e a mesma também possui atividades diuréticas, antirreumáticas, e ajuda no tratamento de cefaleias e ácido úrico (RIBEIRO M, et al., 2004). Segundo a Organização Pan- Americana de Saúde (OPAS), 2017 cerca de 7,4 milhões de pessoas faleceram devido às doenças cardiovasculares. Um dos fatores comuns que prejudica os indivíduos com problemas cardiovasculares é o nível elevado de colesterol. O colesterol é um esteroide presente em quase todos os tecidos, inclusive no sistema nervoso onde faz parte da composição da mielina. É um componente importante das membranas celulares e apresenta-se como precursor de ácidos biliares, da vitamina D e de hormônios esteroidais (LUDKE MCMM e LÓPEZ J, 1999). Grande parte do colesterol presente no organismo é proveniente da síntese no fígado e intestino, já a outra parte é proveniente de dietas.

A presença em maior quantidade de colesterol no sangue pode desencadear uma série de danos, um exemplo é seu acúmulo nas paredes dos vasos sanguíneos definido como aterosclerose. Para ser transportado pelo sangue é necessária a presença das lipoproteínas, que são formadas por lipídios e 
proteínas. O dente-de-leão possui propriedades que podem contribuir com a prevenção e até mesmo no tratamento contra essas patologias. Estudos demonstram um aumento da concentração de HDL (High Density Lipoprotein, ou Lipoproteína de Alta Densidade, em português) com o uso desta planta (CHOI U, et al., 2010). O HDL remove o excesso de colesterol dos tecidos, guiando-o para o fígado, onde é degradado. O processo realizado pelo HDL pode ser definido como transporte reverso.

Dentro deste contexto, o objetivo desta revisão de literatura foi uma revisão integrativa da literatura abrangendo estudos com base em evidências com experimentos in vivo e in vitro para avaliação dos efeitos fitoterápicos do dente-de-leão, que buscaram comprovar os seus usos tradicionais.

\section{MÉTODOS}

Quais são os usos fitoterápicos tradicionais da planta Taraxacum officinale, Dente-de-leão, que foram pesquisados cientificamente? Foi realizada uma busca em meio eletrônico utilizando Google Acadêmico, com auxilio das bases de dados da Scientific Eletronic Library Online (Scielo), Literatura Latino-Americana e do Caribe em Ciências da Saúde (Lilacs) e do The Journal of Clinical Investigation (JCl), no período de Agosto a Setembro de 2019.

Os descritores de pesquisa utilizados foram: "Dente-de-leão" E "etnomarfacologia", "propriedades fitoterápicas" OU "Taxaracum officinale", bem como seus correspondentes em língua inglesa. A lista inicial de artigos foi submetida a análise por dois autores, que aplicaram critérios de inclusão e exclusão para determinar a amostra final do artigo. Foram incluídos artigos que abordassem o tema com experimentos "in vivo" e/ou "in vitro" referentes aos efeitos biológicos do dente-de-leão, relacionados aos seus efeitos descritos nos usos tradicionais. O critério de exclusão foi realizado a partir de estudos que não obtiveram relatos associados aos usos tradicionais e propriedades fitoterápicas do dente-de-leão e artigos publicados antes de 2004.

Figura 1 - Critérios de exclusão e inclusão de artigos.

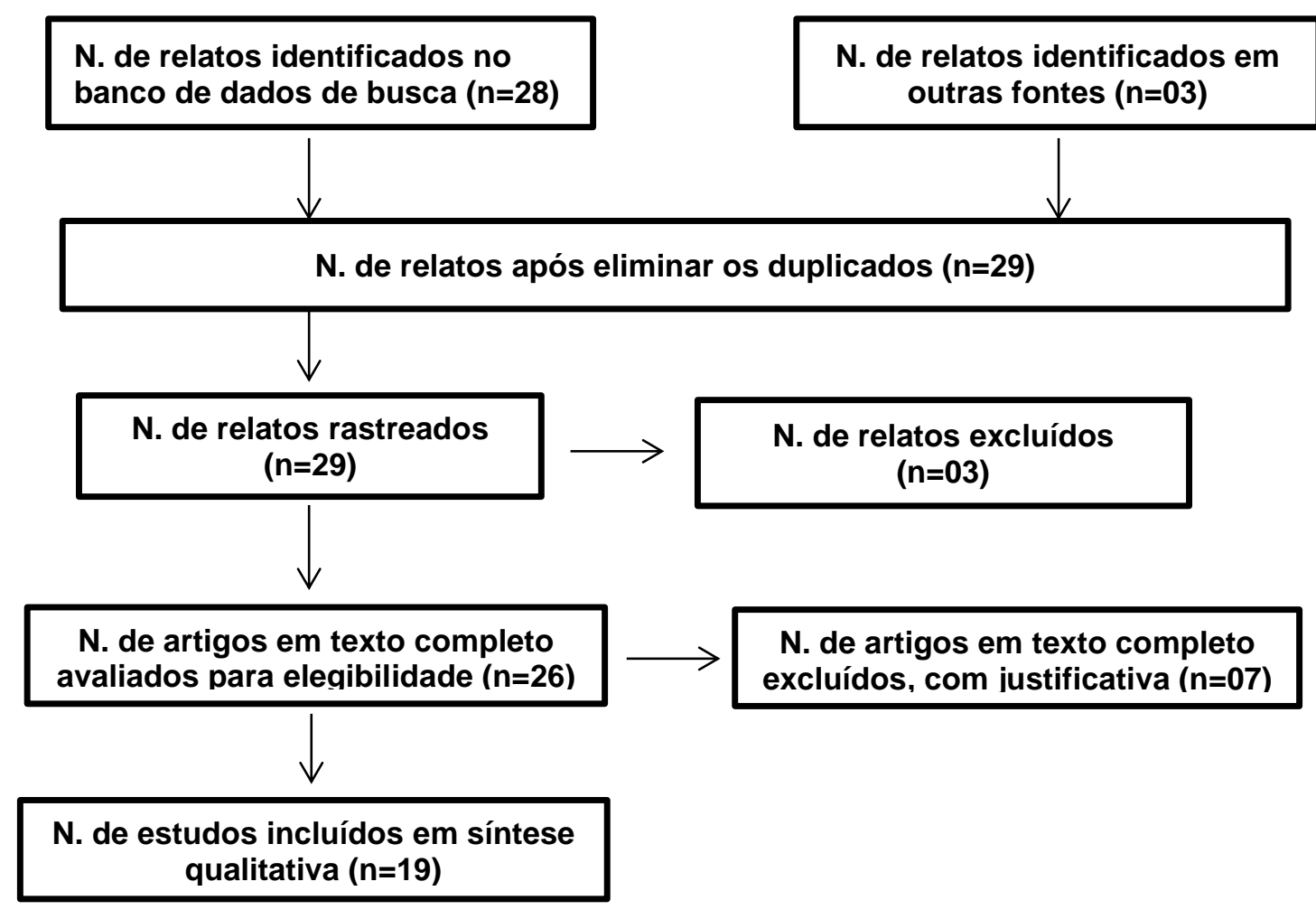

Fonte: Oliveira GMS, et al., 2019. 


\section{RESULTADOS E DISCUSSÃO}

Para o estudo foram encontrados 25 artigos, sendo que após a aplicação do processo de seleção de inclusão e exclusão, foram selecionados 19 artigos, dentre eles revisões, estudos clínicos e experimentais (Quadro 1).

Quadro 1 - Tipos de estudos e principais resultados dos artigos.

\begin{tabular}{|c|c|}
\hline 1 & $\begin{array}{l}\text { el, C e Busia, K (2005) - citação indireta, os autores abordam sobre a utilização da planta para o } \\
\text { tamento da hipertensão. }\end{array}$ \\
\hline 2 & $\begin{array}{l}\text { Caraballo, et al. (2004) - citação indireta, é referida a utilização da planta para o tratamento da } \\
\text { malária. }\end{array}$ \\
\hline 3 & $\begin{array}{l}\text { Carrasco BG, et al. (2015) - citação indireta, os autores se basearam em experimentos in vivo, } \\
\text { comprovando a eficacia do dente-de-leão sobre o aumento de Lipoproteína de Alta Densidade (HDL) } \\
\text { na circulação. }\end{array}$ \\
\hline 4 & $\begin{array}{l}\text { Choi U, et al. (2010) - citação indireta, o artigo foi baseado em experimentos in vivo, para a } \\
\text { comprovação dos efeitos hipolipidêmicos, do qual obteve resultado positivo. }\end{array}$ \\
\hline 5 & $\begin{array}{l}\text { Guarrera, PM; SAVO, V (2013) - citação indireta, os autores } \\
\text { aplicação terapêutica do Taraxacum officinales. }\end{array}$ \\
\hline 6 & $\begin{array}{l}\text { Guiracocha, JCM (2014) - citação indireta, o autor através de teste in vivo e in vitro, comprova os } \\
\text { efeitos positivos em relação á melhora de transtornos hepáticos. }\end{array}$ \\
\hline 7 & $\begin{array}{l}\text { Lima, KSP (2011) - citação indireta, onde o autor ressalta sobre a capacidade das vitaminas A e C } \\
\text { em proteger o organismo de radicais livres. }\end{array}$ \\
\hline 8 & $\begin{array}{l}\text { Ludke MCMM, López, J (1999) - citação indireta, explica sobre o colesterol estar presente em quase } \\
\text { todos os tecidos, sendo responsavel como precursor de ácidos biliares, vitamina D e hormônios } \\
\text { estereoidais. }\end{array}$ \\
\hline 9 & dente-de-leão, podendo ser utilizada de vá \\
\hline 10 & alívio de dor, sendo usado \\
\hline 11 & $\begin{array}{l}\text { Oliveira, B; Santos, J (2018) - citação indireta, através de experimentos in vivo e in vitro foi } \\
\text { comprovada a existência de atividade antioxidante da Taraxacum officinales. }\end{array}$ \\
\hline 12 & $\begin{array}{l}\text { OPAS - Organização Pan - Americana da Saúde (2017) - citação indireta, cita sobre o nível elevado } \\
\text { de colesterol, que apresenta como principal causa de falecimentos devido problemas } \\
\text { cardiovasculares. }\end{array}$ \\
\hline 13 & Ribeiro, M, et al. (2004) - citação indireta, explica sobre a \\
\hline 14 & $\begin{array}{l}\text { Rigoberto, JVH (2017) - citação indireta, afirma sobre a capacidade da planta medicinal melhoras } \\
\text { problemas hepatotóxicos. }\end{array}$ \\
\hline 15 & $\begin{array}{l}\text { Roque, N; Bautista, } \mathrm{H} \text { (2008) - citação indireta, fala sobre as principais ações farmacológicas, suas } \\
\text { utilizações e componentes dos quais a planta é rica. }\end{array}$ \\
\hline 16 & $\begin{array}{l}\text { Rufino, MSM, et al. (2007) - citação indireta, explica sobre a determinação do potencial antioxidante } \\
\text { contido na planta. }\end{array}$ \\
\hline 17 & $\begin{array}{l}\text { Shutz, K, et al. (2006) - citação indireta, ressalta sobre a utilização da planta no tratamento de } \\
\text { diarréias, gota e baço. }\end{array}$ \\
\hline 18 & $\begin{array}{l}\text { Sweeney, B, et al. (2005) - citação indireta, fala sobre a utilização da raiz da planta para tratar } \\
\text { doenças nos rins, azia e dispepsia. }\end{array}$ \\
\hline 19 & vres. \\
\hline
\end{tabular}

Fonte: Oliveira GMS, et al., 2019. 
A primeira referência sobre a espécie vegetal Taraxacum officinale F.H. Wigg., data dos séculos $\mathrm{X}$ e XI, na qual associa esta planta ao tratamento de doenças hepáticas e relacionadas ao baço. Mais tarde, o alemão Fuchs (1543), referencia a sua utilização no tratamento de diarreia, bolhas, gota e queixas a nível hepático e do baço (SCHÜTZ K, et al., 2006). Já, na América do Norte, os aborígenes utilizavam infusões e decocções da raiz da planta para tratar doenças nos rins, azia e dispepsia (SWEENEY B, et al., 2005). Na Venezuela, é referida a sua utilização para o tratamento da malária (CARABALLO A, et al., 2004), enquanto que no Gana abordam as suas potencialidades no tratamento da hipertensão (ABEL C e BUSIA K, 2005). Houve a abordagem de Mustafa B, et al., (2012), dando ênfase nos usos tradicionais na região do Kosovo. A infusão produzida a partir das flores de dente-de-leão era utilizada nesta região para alívio da dor de estômago, inflamação do sistema urinário e respiratório e dores menstruais. Enquanto que as suas folhas eram utilizadas também para as dores de dentes quando mastigadas durante alguns minutos e para distúrbios pulmonares quando em infusão. Na Itália, o dente-de-leão comum é referenciado como uma das plantas com maior diversidade de aplicações terapêuticas, nas quais se incluem propriedades colagogas, analgésicas, digestivas, antirreumáticas, hipocolesterolemiante, diuréticas, laxativas e eupépticas (GUARRERA PM e SAVO V, 2013).

Como pode se observar, há muitos relatos de comprovação da eficácia medicinais desta planta, e diversos se dão pelas referências geradas na sua utilização popular, deste modo, pode-se verificar que seu emprego se deve mais ao uso tradicional do que por provas científicas destas potencialidades, apesar de já existirem diversos artigos científicos, desde o século XIX, sobre pesquisas das atividades terapêuticas do dente-deleão. Apesar de não apresentarem os métodos explícitos nos artigos científicos publicados, de acordo com Rigoberto JVH (2017) e Guiracocha JCM (2014), as análises in vivo em ratos, realizadas com a Taraxacum officinale, onde foram obtidos resultados positivos sobre o mesmo em relação aos transtornos hepáticos. Rigoberto JVH (2017) ainda afirma que pelas análises em animais com extrato etanólico de dente de leão, resultou em êxito considerável reduzindo os efeitos e proporcionando a melhora de hepatotoxicidade induzida por tetracloreto de carbono (CC14), posteriormente durante estudos e conclusões histopatológicas observouse que os efeitos de melhora causados eram devido a presença de diferentes substâncias fitoquímicas no extrato.

Oliveira B, et al., (2018), por ensaios in vivo e in vitro, comprovaram a existência de atividade antioxidante da planta Dente-de-leão, possuindo a ação de prorrogar ou inibir danos oxidativos, sendo rica em vitaminas A e C, com isto, mantendo a capacidade de proteger o organismo de radicais livres e espécies reativas de oxigênio. que se trata de uma forma reduzida de oxigênio da qual tem por sua finalidade reagir juntamente com outras substâncias resultando em diversas reações, como oxidação de componentes celulares, podendo levar à morte celular (LIMA KSP, 2011), acarretando alterações funcionais, como até mesmo o desenvolvimento de patologias (TURECK C, et al., 2017). Para a determinação do potencial antioxidante contido na planta, utilizou-se o extrato e cálculos com a preparação de solução de 2,2-difenil-1-picrilhidrazilo (DPPH) em extração a quente e extração a frio, segundo (RUFINO M S M et. al., 2007) onde se pode observar a máxima absorção para estabelecer o poder antioxidante através de correções lineares. Se a amostra obteve um maior consumo de DPPH expressa maior atividade antioxidante, melhorando sua capacidade de absorver radicais livres.

Os artigos utilizados para a comprovação dos efeitos hipolipidêmicos do de dente-de-leão foram selecionados com experimentos in vivo e in vitro. $\mathrm{O}$ artigo com base em experimentos in vivo, realizado pelo pesquisador Chol U, et. al., (2010), utilizou 20 coelhos brancos da Nova Zelândia (com 16 semanas e entre $1,8-2,2$ quilogramas $(\mathrm{kg})$. Foram divididos em 4 diferentes grupos: normal, controle, dente-de-leão e raiz suplementada. Durante o estudo se mantiveram em gaiolas individuais, em sala com ar condicionado $\left(19^{\circ} \mathrm{C}\right.$ $\pm 1^{\circ} \mathrm{C}, 55 \pm 5 \%$ de umidade), com ciclos de claro e escuro por 12 horas. Todos os grupos foram alimentados por uma dieta normal por uma semana. Após este período, os diferentes grupos foram alimentados com uma dieta hipercolesterolêmica contendo ração de coelho, enriquecida com $1 \%$ do peso por peso(p/p) de colesterol. Apenas o grupo denominado de normal não foi submetido a esta alimentação. Subsequentemente, foram divididos aleatoriamente em um grupo hipercolesterolêmico $(n=7,1 \%$ de colesterol elevado) e dois grupos de tratamento $(n=7,1 \%$ de colesterol elevado juntamente com $1 \%$ das folhas de dente-de-leão e raiz 
respectivamente). A ingestão de alimento foi padronizada em $250 \mathrm{~g} /$ dia para cada coelho durante as 4 semanas do experimento. Este experimento foi realizado em instalações aprovadas pela Associação para Avaliação de Cuidados com Animais de Laboratórios (AAALAC) Internacional. Todos os procedimentos foram aprovados pelo Comitê Institucional de Laboratórios e Uso Animal (IACUC) em conformidade (CHOI U, et al., 2010).

De acordo com os resultados obtidos por Choi U, et al., (2010), sobre a utilização do dente-de-leão como hipolipemiante, tal ação foi comprovada na pesquisa realizada. Foram utilizados testes in vivo com coelhos, sendo obtido sucesso no aumento considerável de HDL na circulação, e deste modo diminuindo o valor de LDL, evitando as placas de ateroma em veias e artérias dos coelhos. O segundo artigo foi baseado em experimentos in vivo, realizado pelo pesquisador Carrasco et. al. (2015), utilizando culturas de células através de pré-adipócitos de células oriundas de embriões de camundongos suiços extraídas prematuramente (3T3L1). As células foram cultivadas em meio Eagle, modificado por Dulbecco suplementado com $10 \%$ de FBS, além de 10\% (v/v) de soro bovino fetal, solução tampão de ácido 4-(2-hidroxietil)-1piperazinoetanossulfórrico 25 milímetros $(\mathrm{mM}), 1 \%(\mathrm{v} / \mathrm{v})$ de mistura antibiótico-antimicótica a $37^{\circ} \mathrm{C}$ em atmosfera umidificada com $5 \%$ de dióxido de carbono $\left(\mathrm{CO}^{2}\right)$. Dois dias depois de atingir a confluência, iniciou-se a diferenciação pela adição de agentes adipogênicos (insulina 5 microgramas por mililitros $(\mu \mathrm{g} / \mathrm{ml}$ ), desametasona (Dex) 0,25 micrometro $(\mu \mathrm{M})$ e IBMX 0,5 milímetros(mM)) ao meio de cultura de Dulbecco MEM (DMEM) mais $10 \%$ de soro fetal bovino(FBS). Após 3 dias, o meio foi removido e as células foram mantidas em DMEM mais $10 \%$ de meio FBS e $10 \mu \mathrm{g} / \mathrm{ml}$ de insulina até a utilização, geralmente 1 a 3 dias após a conclusão da diferenciação. A coloração Oil-Red-O dos adipócitos 3T3-L1 foi realizado como descrito anteriormente. Todas as experiências foram realizadas com 10 dias após a diferenciação.

Segundo Rigoberto JVH (2017), após análises e testes em ratos, observou-se a melhora no funcionamento do fígado em diversos tipos de transtornos hepáticos com o uso de extratos do dente-de-leão. Ainda pelos testes in vivo e in vitro houveram efeitos fitoterápicos contra danos hepáticos causados pelo uso de álcool e, também apresentou um grande potencial de prevenção contra hepatotoxicidade provocado pelo mesmo (GUIRACOCHA JCM , 2014). Segundo Rufino MSM, et al., (2007), os ensaios realizados sobre o potencial da atividade antioxidante do Taraxacum officinale, realizado em triplicata com a preparação de solução DPPH com extração à quente e a frio, apresentaram atividade antioxidante. Observou-se que a extrato a frio obteve uma eficiência antioxidante menor que o extrato à quente. Essa ação está relacionada com a presença das vitaminas $\mathrm{A}$ e $\mathrm{C}$, que são convertidos em antioxidante ajudando assim a proteger os danos causados pelo estresse oxidativo no organismo (GUIRACOCHA JCM, 2014). Apesar da pesquisa realizada por Carrasco BG, et al., (2015), ser realizado com testes in vitro, foram obtidos, da mesma forma, resultados da eficácia do dente-de-leão sob o aumento de HDL na circulação, além de expor dados mais abrangentes sobre a atuação do mesmo nos radicais livres, os quais também geram danos à saúde humana. Com isso, é confirmado o achado de Choi U, et al., (2010), que cita a capacidade do dente-de-leão na contribuição para prevenção e tratamento contra patologias cardiovasculares provenientes da alta concentração de colesterol na circulação sanguínea.

Apesar dos resultados promissores obtidos, certos pontos sobre o dente-de-leão ainda não foram totalmente esclarecidos de forma satisfatória, requerendo mais investigações futuras. Como exemplo, cita-se as características do dente-de-leão no tratamento e prevenção do câncer. O câncer é um assunto de suma importância na realidade em que o mundo vive hoje. A partir do momento em que surgem novas maneiras para a prevenção e tratamento contra o mesmo, já há chances de melhoria de qualidade de vida para as pessoas que são dependentes de fármacos quimioterápicos. Atualmente, já existem estudos como no Canadá, realizados no Departamento de Química e Bioquímica da Universidade de Windsor, pelo pesquisador Siyaram Pandey (2012), que apontou o dente-de-leão como possível remédio para o câncer.

\section{CONSIDERAÇÕES FINAIS}

Diante dos resultados obtidos através da revisão elaborada sobre os usos tradicionais e propriedades fitoterápicas do dente-de-leão (Taxaracum officinale), ainda há muitas pesquisas que podem reforçar a sua 
ação sob as patologias. Contudo através dos estudos já realizados, obtivemos resultados satisfatórios comprovando a eficácia do dente-de-leão sobre suas atividades antioxidantes no auxílio do estado nutricional da população, a capacidade de proteger e ajudar no tratamento de transtornos hepáticos, e a contribuição que o mesmo apresenta sobre a concentração de HDL no organismo. Podemos observar que o conhecimento cultural da planta ainda é mais presente do que as pesquisas, isso nos remete a importância do desenvolvimento de novos estudos utilizando a Taraxacum officinale.

\section{REFERÊNCIAS}

1. ABEL C, BUSIA K. An Exploratory Ethnobotanical Study of the Practice of Herbal Medicine by the Akan Peoples of Ghana Herbal Medicine in Ghana, Alternative Medicine Review, 10, 2 (2005), p.112-122.

2. CARABALlO A, et al. Preliminary assessment of medicinal plants used as antimalarials in the southeastern, Venezuelan Amazon. Rev. Soc. Bras. Med. Trop. 37, 2(2004). p.186-188.

3. CARRASCO BG, et al. In vitro Hypolipidemic and Antioxidant Effects of Leaf and Root Extracts of Taraxacum officinale, Madri. Espanha, 2015; 3(2), 38-54.

4. $\mathrm{CHOI} \mathrm{U}$, et al. Hypolipidemic and Antioxidant Effects of Dandelion (Taraxacum officinale) Root and Leaf on Cholesterol-Fed Rabbits, Pohang. Coréia, 2010; 11 (1), 67-78.

5. GUARRERA PM, SAVO V. Perceived health properties of wild and cultivated food plants in local and popular traditions of Italy: A review. Journal of Ethnopharmacology. 146, 3(2013). p.659-680.

6. GUIRACOCHA JCM. Estudio Bibliográfico de las Propiedades Medicinales y Nutricionales del Diente de León., Universidad Católica de Cuenca, Cuenca (2014) p. 34- 42.

7. LIMA KSP. Avaliação da Actividade Antioxidante e Antimutagénica em Diferentes Infusões Medicinais: Barbas de Milho, Carqueja, Dente de Leão, Folhas de Oliveira e Urtiga-Branca, Universidade Nova de Lisboa, Setembro (2011) p. 10-19.

8. LUDKE MCMM, LÓPEZ J. Colesterol e Composição dos Ácidos Graxos nas Dietas para Humanos e na Carcaça Suína, Ciência Rural, Santa Maria, v. 29, n. 1, p.181-187, 1999;

9. MARTINEZ M, et al. Taraxacum officinale and related species - An ethnopharmacological review and its potential as a commercial medicinal plant. Journal of Ethnopharmacology, 169, 1(2015), p.244-262.

10. MUSTAFA B, et al. A. An ethnobotanical survey of the Gollak region, Kosovo. Genetic Resoures and Crop Evolution 59(2012), p.739-754.

11. OLIVEIRA B, SANTOS J. Estudo das Propriedades da Taraxacum officinale (Dente de Leão), Universidade do Vale do Paraíba, São José dos Campos (2018) p. 29-31.

12. OPAS- Organização Pan-Americana da Saúde. Doenças cardiovasculares. Brasília (DF); 2017.

13. RIBEIRO M, et al. Taraxacum officinale WEBER (Dente De Leão). Uma Revisão Das Propriedades e Potencialidades Medicinais. Núcleo de pesquisas de Agricultura (NUPELLA). Departamento de Biologia da Universidade Estadual de Maringá. Maringá (2004).

14. RIGOBERTO JVH. Efecto del Extracto de Diente de León (Taraxacum officinale), Sobre el Comportamiento Productivo y Enzimas Hepáticas Séricas en Pollos de Engorde., Universidad Técnica de Ambato, Ambato (2017) p. 2-18.

15. ROQUE N, BAUTISTA H. Asteraceae Caracterização e Morfologia Floral., Universidade Federal da Bahia, Salvador (2008) p. 11-19.

16. RUFINO MSM, et al. Metodologia Científica: Determinação da Atividade Antioxidante Total em Frutas pela Captura do Radical Livre DPPH. Fortaleza (2007).

17. SCHÜTZ K, et al. Taraxacum - A review on its phytochemical and pharmacological profile. Journal of Ethnopharmacology, 107, 3(2006), p.313-323.

18. SWEENEY B, et al. Evidence-Based Systematic Review of Dandelion (Taraxacum officinale). Natural Standard Research Collaboration, 5, 1(2005), p.79-93.

19. TURECK C, et al. Avaliação da Ingestão de Nutrientes Antioxidantes Pela População Brasileira e Sua Relação Com o Estado Nutricional. Revista Brasileira de Epidemiologia, São Paulo (2017). 